\title{
La recreación del universo profesional en el aula a través de técnicas de aprendizaje colaborativo: el Método del Caso y el Juego de Rol ${ }^{1}$
}

\author{
María Jesús RuIZ-MuÑoz \\ Universidad de Málaga \\ mariajesus@uma.es \\ Isabel RuIz-MoRA \\ Universidad de Málaga \\ isabelruiz@uma.es
}

\begin{abstract}
Resumen:
En el presente trabajo se analiza la aplicación del Método del Caso y del Juego de Rol en las titulaciones de Publicidad y Relaciones Públicas y de Comunicación Audiovisual, respectivamente. Se trata de profundizar en el desarrollo de metodologías docentes que propicien la adquisición de competencias profesionales y transversales mediante el trabajo colaborativo. Los resultados de la evaluación de estas actividades nos trasladan la disposición favorable del alumnado hacia la utilización de técnicas y herramientas pedagógicas que permitan recrear el contexto de trabajo profesional.
\end{abstract}

Palabras Clave: Método del Caso; Juego de Rol; competencias profesionales; trabajo colaborativo; estudios de Comunicación.

\section{The recreation of professional world into the classroom through collabora- tive learning techniques: the Case Method and Role Playing}

\begin{abstract}
:
This paper discusses the application of Case Method and Role Playing in the degrees of Advertising and Public Relations and Communication Studies, respectively. We try to further in the development of teaching methods that promote the acquisition of professional and cross-curricular skills through collaborative work. The results of the evaluation of these activities show us the willingness of students to use the techniques and tools that recreate the context of professional work.
\end{abstract}

Key Words: Case method; Role play; professional skills; collaborative work; Communication Studies.

Referencia normalizada:

Ruiz Muños, M. J. y Ruiz Mora, I. (2014): La recreación del universo profesional en el aula a través de técnicas de aprendizaje colaborativo: el métdod del caso y el juego del rol. Historia y Comunicación Social. Vol. 19. Núm. Especial Febrero. Págs. 223-234

Sumario: 1. Introducción: El método del Caso y el Juego de Rol como herramientas para el desarrollo de competencias profesionales y transversales. 2. Metodología. 3. El Método del Caso en el proceso de enseñanza-aprendizaje de las Relaciones Públicas. 4. El Juego de Rol en el proceso de enseñanza-aprendizaje de Comunicación Audiovisual. 5. Conclusiones. 6. Referencias bibliográficas. 


\section{Introducción: el método del caso y el juego del rol como herramientas para el desarrllo de competencias profesionales y transversales.}

El Método del Caso (MdC) o estudio de caso fue introducido por primera vez en 1870 por Christopher C. Langdell en una de sus clases en la Harvard Law School, poniendo así de relieve la importancia del estudio de situaciones reales y la obtención de conclusiones propias (Hernández Andreu y García Ruiz, 2010: 1). En 1920, esta metodología llegó al Harvard Business School y desde ahí se expandió por todas las escuelas de negocios. Para Ruiz Campo (2011: 5), el objeto del MdC es

(...) formar, más que informar, gracias a que da importancia tanto a los contenidos conceptuales de la materia, como al proceso de aprendizaje, que debe ayudar a los alumnos a desarrollar la capacidad de enfocar los problemas y afrontar las situaciones nuevas con criterios propios. Es decir, transmite las capacidades de enfrentarse a situaciones nuevas con los conocimientos recibidos.

Por otro lado, como señala Ruiz Campo (2011: 9), el MdC permite atraer el interés de los alumnos, involucrarlos en la asignatura, despertar en ellos inquietudes y ofrece un valor añadido al aprendizaje, puesto que favorece la retención y la aplicación de conocimientos. En cuanto a la principal desventaja de esta herramienta, cabe subrayar que es el autor el que tiene que desarrollar la principal tarea de investigación y de recopilación de datos; este modelo es un complemento a la formación teórica y, por tanto, no la sustituye sino que sencillamente sería un valor añadido. Además, deben tenerse en cuenta las dificultades que entraña redactar correctamente un caso para que éste finalmente resulte efectivo.

En el presente trabajo también se ha considerado la aplicación del Juego de Rol (JdR) como "una técnica de enseñanza-aprendizaje mediante la que, de un modo colaborativo, los alumnos aprenden jugando y experimentando y el profesor enseña reforzando el aprendizaje" (Ortiz de Urbina et al, 2010: 284). Para Imbernón Muñoz y Medina Moya (2008: 25-26), el JdR es un proceso que posibilita "revivir una situación que nos permita comprender el porqué de las situaciones y actitudes de los demás, a partir de pequeñas representaciones".

Esta técnica es muy válida para ayudar a entender un problema y desarrollar la empatía, la posibilidad de ponernos y comprender la situación de los demás, por lo que favorece la cohesión del grupo. Es una de las actividades más indicadas para saber defender ideas que no necesariamente coinciden con la propia. (Ortiz de Urbina et al., 2010: 284)

No obstante, conviene matizar que sin una correcta distribución de los roles entre el alumnado y sin una constante retroalimentación por parte del profesor, lo cual no siempre es posible y además requiere una gran dedicación, el JdR puede perder gran parte de su efectividad.

Trabajar con el MdC y el JdR nos va a permitir profundizar en las diferencias sustanciales entre ambas herramientas (Imbernón Muñoz y Medina Moya, 2008: 28). En primer lugar, en el JdR, el hecho de que los estudiantes tengan que asumir 
un papel está orientado a despertar emociones y nuevas formas de comportamiento mientras que, en el MdC, se trata de pensar analíticamente, de separar lo esencial de lo secundario y de detectar las prioridades. Por otra parte, el MdC requiere, en numerosas ocasiones, que el alumnado posea conocimientos previos de la asignatura en la que se trabaja, por lo que este instrumento resulta más indicado para estudiar una materia nueva trabajando con una situación simulada.

En general, en el contexto universitario hoy en día, el docente-mediador debe ser consciente de que estas herramientas encaminadas al aprendizaje colaborativo resultan sumamente apropiadas tanto para el desarrollo de competencias profesionales como para reforzar la adquisición de competencias transversales. Asimismo, se revela como algo fundamental la búsqueda de planteamientos que resulten motivadores para el alumnado y por tanto, para su aprendizaje.

(...) el aprendizaje colaborativo no sólo sirve para fomentar el interés y la implicación del alumnado sino que también resulta útil para trabajar otras competencias transversales que se relacionan con alimentar su capacidad de análisis y promover el trabajo con nuevas tecnologías. Mediante el trabajo colaborativo, desarrollamos entre los estudiantes algo más que competencias profesionales, ya que provocamos en ellos un pensamiento más crítico y solidario. (Ruiz Mora et al, 2012: 846)

En el Espacio Europeo de Educación Superior (EEES), la introducción del aprendizaje por competencias ha traído consigo considerables transformaciones en el proceso de enseñanza-aprendizaje, puesto que se le otorga al alumno un papel activo, convirtiendo así al docente en un mediador para el desarrollo de actitudes, destrezas, habilidades y conocimientos. En palabras de Ruiz Mora y Olmedo Salar (2012: 742), se están "aplicando nuevas metodologías de enseñanza y aprendizaje enfocadas a propiciar nuevos entornos en las aulas, en los que los estudiantes son protagonistas de su propio aprendizaje y el docente se convierte en catalizador de las nuevas experiencias y del aprendizaje".

Estos cambios en las metodologías docentes han evolucionado en paralelo a la sociedad actual, en la que se imponen cada vez más las formas de trabajo colaborativo "tratando de beneficiar al grupo compartiendo aportaciones y conocimiento, en contextos académicos y laborales" (Sáez López y Ruiz Ruiz, 2011: 118). Así pues, no debe extrañarnos que el nuevo modelo educativo resulte mucho más permeable a las exigencias de un mundo profesional cada vez más flexible y complejo, en el que se requiere potenciar la capacidad para trabajar en equipo, la asertividad y el liderazgo.

En lo que respecta a los estudios de Comunicación, destacan por su marcado carácter práctico, puesto que uno de los objetivos primordiales en el proceso de enseñanza-aprendizaje es la asimilación de las rutinas profesionales. La actividad de los futuros profesionales de la Comunicación se desarrolla en entornos de trabajo grupal y, por ello, las metodologías aplicadas en el aula deberán incidir en la implementación de dinámicas orientadas al aprendizaje colaborativo basado en casos reales. En este sentido, también es sumamente enriquecedor el uso del campus virtual puesto que favorece el aprendizaje activo, posibilita el intercambio de ideas y proporciona 
un feedback en tiempo real entre los estudiantes, incentivando así el espíritu crítico y la toma de decisiones conjuntas. En general, el uso de herramientas TIC en aras del aprendizaje colaborativo no sólo permite reforzar la adquisición de competencias profesionales y transversales sino también mejorar la interacción entre el profesorado y el alumnado, tanto desde una enseñanza presencial como virtual (Cuadrado Gordillo et al, 2013:14).

\section{Metodología}

El trabajo planteado ha tenido como objetivo fundamental conocer qué opinan los estudiantes sobre las metodologías docentes que propician un aprendizaje a través de la práctica con casos reales. Como objetivo secundario, nos proponemos diseñar actividades docentes que sean atractivas para los estudiantes y que permitan la adquisición de competencias profesionales mediante el aprendizaje colaborativo.

Con el fin último de alcanzar los objetivos propuestos y tomando como referencia el estudio de Ruiz Mora et al. (2012: 846), donde se ofrece un listado de las herramientas docentes que favorecen la consecución de competencias profesionales, hemos seleccionado el MdC y el JdR para aplicarlos en el aula. También se han tenido en consideración otras experiencias de trabajo con competencias profesionales que pueden considerarse un antecedente en el ámbito de las Ciencias de la Comunicación (Monedero, 2011: 53-66).

El diseño metodológico que ha guiado el presente trabajo, desarrollado en la Facultad de Ciencias de la Comunicación de la Universidad de Málaga, se ha concretado en tres fases:

1. Creación. En función de las asignaturas y de las competencias de cada una, hemos adjudicado el MdC a Dirección Estratégica en Relaciones Públicas, de $2^{\circ}$ curso del Grado de Publicidad y Relaciones Públicas, y el JdR a Narrativa Audiovisual, de $3^{\circ}$ curso de la Licenciatura en Comunicación Audiovisual. Para cada asignatura se ha diseñado una actividad basada en las metodologías descritas, teniendo en cuenta el temario y los objetivos. Igualmente se han generado documentos ad hoc y se han puesto a disposición de los estudiantes otros recursos que les ayudasen en el desarrollo de la actividad.

2. Aplicación. Ejecución de las actividades en el aula.

3. Evaluación. Diseño de dos cuestionarios para la evaluación de cada actividad por parte de los estudiantes participantes que nos permitirán la extracción de conclusiones. 


\section{El método del caso en el proceso de enseñanza-aprendizaje de las relaciones públicas}

El desarrollo del MdC en la asignatura Dirección Estratégica en Relaciones Públicas se ha trabajado a través del caso de la multinacional Nestlé y de la crisis comunicativa a la que se enfrentó en el año 2010. Nos encontramos ante un caso real, que se produce en el ámbito de actuación de las Relaciones Públicas en las organizaciones y que parte de un problema (una crisis) al que los estudiantes deben dar una solución. En total, han participado 53 alumnos y alumnas.

El origen de la crisis surgió cuando la organización Greenpeace publicó un informe que acusaba a Nestlé de utilizar aceite de palma procedente de selvas deforestadas de Indonesia para elaborar sus productos. Esta situación se vio agravada porque la multinacional, a través de la compra de este producto a su proveedor local, estaba contribuyendo a la destrucción del hábitat de los orangutanes como especie protegida. La crisis surgió cuando Nestlé rechazó cualquier responsabilidad e hizo oídos sordos a las demandas de toda la sociedad.

Específicamente, con esta actividad se ha pretendido que los alumnos valoren el trabajo en equipo como propuesta para la solución de problemas y que sean capaces de identificar la importancia de la correcta gestión de las relaciones con los públicos con un caso real.

El MdC aplicado en el aula ha consistido en una actividad evaluable llevada a cabo en los grupos reducidos de la asignatura y se ha tenido en cuenta tanto la participación en los debates en clase como la resolución del caso y la defensa del mismo en la evaluación.

La primera parte de la actividad se basó en la presentación del caso, la forma de trabajo que se iba a seguir y la subida del material necesario al campus virtual. Con un fin informativo, se pretendía que los alumnos tuvieran una documentación completa del caso, lo que les facilitaría su participación en clase y el trabajo en equipo.

Posteriormente se pasó a la segunda fase, centrada en los debates en clase, donde los alumnos expusieron sus opiniones ante el resto de compañeros, las posibles soluciones al caso y las razones que podrían argumentar las posiciones defendidas por Nestlé y Greenpeace. En esta fase, se produjo una interacción en el Grupo Grande. La tercera fase giró en torno al trabajo en equipo y, en ella, los estudiantes realizaron la actividad como si fueran una agencia de Comunicación que debía ofrecer una alternativa/solución al problema que se les presentaba. Trabajaron desde el punto de vista profesional de la asesoría. La última fase consistió en la entrega del caso y en su posterior defensa en clase, donde tanto los alumnos como la profesora ofrecían un feedback.

La evaluación de la actividad se ha llevado a cabo entre los estudiantes participantes que, de forma voluntaria y anónima, han valorado el MdC en el aula. Un $71 \%$ de las personas sondeadas eran mujeres y un $29 \%$, hombres, ambos con edades 
comprendidas entre 19 y 31 años. A continuación, presentamos los datos sobre la evaluación de la actividad, la metodología empleada y el aprendizaje colaborativo, a través de preguntas abiertas y cerradas:

- A la pregunta referente al nivel de satisfacción con el empleo del MdC en clase, un $53 \%$ manifiesta estar muy satisfecho; un $41 \%$, satisfecho y un $6 \%$, algo satisfecho. En ningún caso encontramos respuestas que aludan a que el estudiante está poco o nada satisfecho.

- Cuando le pedimos a los estudiantes su opinión sobre la inclusión de metodologías en el aula, como el MdC, vinculadas con el mundo profesional de las Relaciones Públicas y que trabajan con casos reales, todos ofrecen una opinión positiva sobre la aplicación del MdC. Un 35\% conoce la metodología porque ha trabajado con ella anteriormente y para un $65 \%$ se trata de una novedad. En un $100 \%$ de los casos, los participantes volverían a trabajar con esta metodología e igualmente, el $100 \%$ considera que sería beneficioso utilizar esta actividad en otra asignatura.

- Al plantearle al alumnado que exprese cómo valora el trabajo colaborativo asociado al MdC, como los debates, el trabajo en equipo o las presentaciones, nos ofrece diferentes respuestas. Un 12\% presenta algún problema relacionado con la actividad, principalmente relacionado con el tiempo de trabajo que conlleva y con los problemas de compartir tarea con personas que se implican menos. Un $88 \%$, por el contrario, valora positivamente el trabajo colaborativo porque le acerca a las formas de realizar las tareas que encontrarán en las organizaciones, les ayuda a clarificar dudas, los familiariza con los procesos de búsqueda de consenso y respeto de ideas diferentes, mejora su involucración con la actividad y su capacidad para desarrollar una argumentación sólida que defienda sus posturas.

En cuanto a los aspectos positivos, encontramos que los estudiantes consideran que el MdC les ayuda a:

- Comprender el mundo que les rodea, ya que se enfoca en problemas reales.

- Potenciar su participación en la actividad, a través de una mayor implicación que les lleva a razonar más y mejor el caso.

- Propicia un mayor trabajo práctico: "nos vamos familiarizando con los casos reales aparecidos en la comunicación, siendo más fácil en nuestro futuro reconocer esos mismos fallos y poder evitar que se lleven a cabo".

Nos permitimos también ofrecer una afirmación de un estudiante reflejada en el cuestionario de evaluación, en la que se recoge el principal beneficio que el alumnado ha detectado en esta metodología: "está orientado a aprender verdaderamente en qué consiste el trabajo de un Relaciones Públicas".

Por otro lado, cuando preguntamos por los aspectos negativos, encontramos las siguientes consideraciones: 
- Diferente nivel de participación en la actividad, coordinación del equipo de trabajo.

- Conocimientos previos insuficientes.

- La dificultad del caso, situaciones en algunas ocasiones desconocidas.

- Falta de práctica.

- Tiempo requerido, necesidad de una amplia investigación para realizar el caso.

- Familiarización con la metodología y los requerimientos.

Las principales quejas de los participantes se relacionan con problemas para organizarse como equipo, distribuir su tiempo y falta de práctica con esta metodología.

Finalmente, solicitamos a los estudiantes que valorasen globalmente la actividad con una puntuación del 1 al 10: un 65\% de ellos le concedió un 8; un 18\%, un 9 y un $18 \%$ un 10. En suma, puede deducirse que la aplicación del MdC ha tenido una buena aceptación entre los estudiantes, obteniendo una calificación global de notable.

\section{El juevo de rol en el preceso de enseñanza-aprendizaje de comunicación audiovisual}

La aplicación del JdR en la asignatura Narrativa Audiovisual ha tenido como objetivo principal el desarrollo de herramientas para la construcción de personajes a través del análisis de los mismos. En cuanto a los objetivos específicos, se ha hecho hincapié en el trabajo en el equipo mediante la recreación de dinámicas propias del contexto profesional y en el desarrollo de la capacidad de síntesis y de las habilidades comunicativas. La actividad aquí descrita también se ha concebido al servicio de que los estudiantes sepan valorar las aportaciones ajenas y aprendan de las críticas, sugerencias y consejos de cara a la revisión y mejora de la redacción y presentación de proyectos audiovisuales.

El JdR que se ha llevado a cabo en el aula, de carácter no evaluable, ha sido diseñado para reforzar las explicaciones de las clases magistrales y para servir de complemento a la preparación del tema sobre construcción de personajes para el examen final. En total, han participado 144 alumnos y alumnas.

En las clases teóricas se estudiaron una serie de elementos que resultan fundamentales para la correcta construcción de personajes en el relato audiovisual: tipo de personaje, caracterización interna, caracterización externa, arco de evolución, relaciones con los otros personajes. Estos aspectos también forman parte de los elementos de la narración que los estudiantes deberían analizar en el examen final de la primera convocatoria ordinaria tras la proyección de un cortometraje de ficción: Sirenas (Fernando León de Aranoa, 1994). 
Además de profundizar en los cimientos de un personaje para determinar si su caracterización global es coherente, matizada y atractiva para el espectador, resulta igualmente necesario que los alumnos trabajen la capacidad de síntesis para poder transmitir de forma eficaz, en general, la esencia de sus proyectos. Por todos estos motivos, seleccionamos la dinámica del pitching, a modo de JdR, con la intención de recrear un contexto óptimo que nos permitiese extraer los puntos clave de la caracterización del personaje y presentarlos de manera sugerente. A grandes rasgos, el pitching es una técnica de venta de productos audiovisuales que está basada en la presentación oral de un proyecto durante un breve intervalo de tiempo que normalmente oscila entre 5 y 10 minutos:

Es como si todo el atractivo de un proyecto pasa por la necesidad de partir de tres frases brillantes con las que ofertarlo e impactar a un interlocutor saturado de propuestas. Es una pura técnica publicitaria, llevada al terreno de las relaciones laborales y la gestación de procesos culturales. Si no partes de un buen eslogan, no vendes. Si no te sabes vender, nadie te compra (García Serrano, 2009: 6).

Para Martínez Abadía (2010: 36), el pitching no sólo permite sintetizar los conceptos fundamentales de un proyecto, sino que requiere el despliegue de habilidades comunicativas y de un grado importante de apasionamiento necesario para que el público se interesen por lo que está explicando un expositor. Así pues, consideramos que la realización de un pitching se revela también como una forma efectiva de motivar al alumnado.

En suma, la actividad planteada ha tenido una duración de dos semanas de clase y ha sido reforzada en las horas de tutoría. La primera fase, dedicada a la introducción y la lectura, se ha llevado a cabo durante cinco días y en ella se presentó la actividad, comentando que se realizaría de forma presencial, con apoyo de material distribuido a través del campus virtual, que se prepararía individualmente para posteriormente realizarse en común y que no sería evaluable. Con el fin de que los estudiantes construyesen de forma esquemática el discurso, se les facilitó a través del campus virtual un resumen con los elementos clave para la construcción y definición de personajes. También se les proporcionó un modelo para la caracterización psicológica de personajes, con la intención de que les resultase útil para familiarizarse con ellos y sacarles partido. En esta etapa contamos con la ventaja de que los alumnos ya estaban familiarizados con la dinámica del pitching a través de la asignatura Teoría y Técnica de la Producción Audiovisual. Aun así, se organizó un repaso en clase para asegurar que habían interiorizado correctamente sus reglas.

La segunda fase, que giró en torno al debate, los ensayos y la resolución de problemas en horario de tutoría, tuvo una duración de ocho días. A lo largo de una semana, se destinaron parte de las clases presenciales y un espacio del campus virtual para revisar el material proporcionado y para plantear ejemplos. La tercera fase consistió en la realización del pitching durante dos días de clase, en el horario de Grupo Grande. Para dotar la actividad de una mayor similitud al contexto profesional, cuatro estudiantes de cada grupo (Grupo A, turno de mañana y Grupo B, turno de tarde), de forma voluntaria, asumieron el rol de panelistas que representaban los intereses 
de producción de: TVE, cadena de televisión pública nacional; Antena 3, cadena de televisión privada nacional; Canal Sur, cadena de televisión pública autonómica y El Deseo, productora cinematográfica fundada por Pedro Almodóvar.

El resto de sus compañeros preparó para la ocasión un personaje popular de ficción cinematográfica o televisiva, siguiendo el esquema indicado en las instrucciones de la práctica, cuyas fortalezas había que defender ante el panel durante un máximo de 5 minutos (si un participante no lograba concluir su discurso en el tiempo estipulado, se cortaba su intervención como sucedería en un pitching real). Al término de cada exposición, los panelistas juzgaron el interés de los respectivos personajes según la línea de producción que representaban (TVE, Antena 3, Canal Sur, El Deseo), formularon preguntas a sus compañeros y compañeras y aportaron sugerencias de cara a la mejora de la construcción de los personajes presentados.

El proceso de evaluación del JdR tuvo lugar el día del examen final de la asignatura. A los estudiantes se les proporción un cuestionario para que valorasen la actividad voluntariamente $\mathrm{y}$, en este sentido, cabe destacar tanto el alto índice de participación del alumnado como que, en todos los casos, la apreciación fuese positiva.

Las estimaciones de los participantes se orientaron fundamentalmente en torno a los siguientes aspectos:-

- Interés de la actividad para repasar el tema sobre personajes.

- Utilidad de preparar los personajes del pitching para resolver mejor la parte de análisis del examen.

- Ventajas de atender a los pitching de los compañeros y compañeras de cara a la parte de análisis del examen.

- Adquisición de conocimientos prácticos para afrontar mejor en el futuro la redacción y presentación de un proyecto audiovisual.

También cabe señalar que se les pidió a los estudiantes que valorasen el material complementario facilitado a través del campus virtual para la realización de la tarea, que pensasen cómo podría mejorarse la actividad y que indicasen cualquier aspecto que considerasen destacable de su experiencia.

A grandes rasgos, lo más relevante para el alumnado, según sus propios comentarios, ha sido que la actividad le ha servido para implicarse más en el estudio de los mecanismos de construcción de personajes y, a su vez, esto le ha resultado útil para llevar a cabo con éxito la parte de análisis del examen. En la parte negativa y en el apartado de sugerencias, los alumnos y alumnas han señalado que las dos horas de pitching de personajes se les hicieron muy cortas y les hubiese gustado que la actividad se prolongase más. En suma, la evaluación revela que la dinámica de JdR ha servido para fomentar el interés y la implicación de los alumnos. 


\section{Conclusiones}

Las metodologías docentes vinculadas al mundo actual, al mundo profesional, poco a poco se van convirtiendo en actividades recurrentes para los docentes con el fin de preparar al alumnado para su incursión en el ámbito laboral. El MdC y el JdR se postulan como dos herramientas que, aplicadas al trabajo en aula, facilitaran la adquisición de competencias profesionales mediante un trabajo colaborativo e inclusivo; sin olvidar la importancia de la actualidad y de la realidad de los casos con los que se practica.

El trabajo con situaciones reales en el aula propicia la adquisición de competencias que aporten a los estudiantes recursos necesarios para poder desenvolverse en el contexto profesional tras su formación universitaria. La resolución de casos basados en problemas y situaciones reales permite mostrar y demostrar a los alumnos cómo están preparados para desarrollar su carrera en adelante.

Adicionalmente, ambas metodologías favorecen una participación activa del alumnado no sólo en el marco de la actividad en sí, sino también en su propio aprendizaje, como hemos comprobado en los resultados expuestos. Son los mismos estudiantes los que descubren cómo su involucración en el proceso de enseñanza repercute positivamente en su aprendizaje.

Los resultados positivos de las actividades y la implicación de los estudiantes nos permiten afirmar que existe una disposición favorable hacia estas metodologías, sobre todo cuando los participantes perciben que tienen un mayor protagonismo en su aprendizaje y éste está íntimamente vinculado con su futuro profesional. Además, esta buena actitud no está necesariamente motivada porque la actividad se asocie a una calificación positiva o negativa. En las dos actividades que se han abordado en el presente trabajo, una de ellas estaba vinculada a la calificación final y la otra no pero, en ambos casos, el resultado ha sido favorable.

Por último, y no por ello menos importante, cabe analizar es el papel que juega el docente en este proceso de enseñanza-aprendizaje. Su labor se torna en fundamental debido a que se hace imprescindible un alto nivel de implicación y de compromiso para el buen funcionamiento de las actividades organizadas en el aula. El docente no sólo debe suministrar el material necesario para el desarrollo de las mismas, sino que también se requiere un trabajo motivador que propicie el intercambio y el diálogo entre los participantes con el fin de alcanzar los objetivos esperados y la implementación de mecanismos que permitan evaluar los resultados que estas metodologías propician en el aprendizaje del alumnado.

\section{Bibliografía}

CUADRADO GORDILLO, I. (et al.) (2013). "Estilos de aprendizaje del alumnado de Psicopedagogía y su implicación en el uso de las TIC y aprendizaje colabora- 
tivo". En: RED. Revista de Educación a Distancia, n 35 , Murcia: Universidad de Murcia. p. 1-19. http://www.um.es/ead/red/35/cuadrado_et_al.pdf (10-09-2013) GARCÍA SERRANO, F. (2009). "Las técnicas del pitching en el mercado audiovisual español: del caramelo al guión". Congreso Internacional Brand Trends. Valencia: CEU Universidad Cardenal Herrera. http://eprints.ucm.es/8577/1/ Pitching_del_caramelo_al_guion.pdf (10-09-2013)

HERNÁNDEZ ANDREU, J. y GARCÍA RUIZ, J. L. (2010). "La aplicación del método del caso a la docencia en Historia de la Empresa". Congreso Internacional IX Encuentro de Didáctica de la Historia Económica. Toledo: Universidad de Castilla - La Mancha. http://www.uclm.es/area/Hinstituciones/IXencuentro HE Toledo/comunicaciones/Sesi\%C3\%B3n\%203b.2\%20JUAN\%20 HERNĀNDEZZ20ANDREU\%20Y\%20JOS\%C3\%89\%20LUIS\%20GARC\%C3\%8DA\%20RUIZ.pdf (06-09-2013)

IMBERNÓN MUÑOZ, F.; MEDINA MOYA, J. L. (2008). Metodología participativa en el aula universitaria. La participación del alumnado. Cuadernos de docencia universitaria 04. Barcelona: ICE y Ediciones Octaedro.

MARTÍNEZ ABADÍA, J. (2010). "Presentación de proyectos audiovisuales". En: Reverberar. Máster Internacional de Comunicación y Educación, Barcelona: Universidad Autónoma de Barcelona. p. 33-36. http://libros.metabiblioteca. org:8080/jspui/bitstream/001/412/8/978-84-693-9778-7.pdf\#page $=34 \quad(10-09$ 2013)

MONEDERO MORALES, C del R. (2011). "Competencias profesionales en continua mutación: el caso de los periodistas y las TIC". En RÍOS ARIZA, J. M. y RUIZ PALMERO, J. (Coords.) (2011). Competencias, TIC e Innovación. Nuevos escenarios para nuevos retos. Sevilla: MAD. p. 53-66.

ORTIZ DE URBINA, M.; MEDINA SALGADO, S.; DE LA CALLE DURÁN, C. (2010). "Herramientas para el aprendizaje colaborativo: una aplicación práctica del juego de rol". En: Teoría de la Educación: Educación y Cultura en la Sociedad de la Información, vol. 11 (núm. 3), Salamanca: Universidad de Salamanca. p. 277-300. http://dialnet.unirioja.es/servlet/articulo?codigo=3610165 (10-09-2013)

RUIZ CAMPO, S. (2011). "Aplicación del EES. Nuevas metodologías: el caso de "el caso". Congreso Internacional de Innovación Docente, Cartagena (Murcia): Universidad Politécnica de Cartagena y Universidad de Murcia. http://repositorio. bib.upct.es/dspace/bitstream/10317/2183/1/c133.pdf (10-09-2013)

RUIZ MORA, I.; RUIZ MUÑOZ, M. J.; GUERRERO NAVARRO, D. (2012). “El desarrollo de competencias profesionales dentro del marco del EEES. El caso de los grados en Comunicación". En: Estudios Sobre el Mensaje Periodístico, $\mathrm{n}^{\mathrm{0}} 18$, Madrid: Universidad Complutense de Madrid. p. 839-847.

RUIZ MORA, I.; OLMEDO SALAR, S. (2012). "Desarrollo de competencias profesionales en el Espacio Europeo de Educación Superior (EEES). Nuevas metodologías en los estudios de Comunicación". En: Revista de Comunicación Vivat Academia, $\mathrm{n}^{\circ}$ Especial Febrero, Madrid: Universidad Complutense de Madrid. p. 739-754. http://pendientedemigracion.ucm.es/info/vivataca/numeros/n117E/ PDFs/Varios26.pdf (10-09-2013) 
SÁEZ LÓPEZ, J. M.; RUIZ RUIZ, J. M. (2011). "Estrategias metodológicas, aprendizaje colaborativo y TIC: un caso en la Escuela Complutense Latinoamericana". En: Revista Complutense de Educación, $\mathrm{n}^{\circ}$ 23, Madrid: Universidad Complutense de Madrid. p. 115-134. http://revistas.ucm.es/index.php/RCED/article/ view/39105/37718 (10-09-2013)

\section{Notas}

1 El trabajo que aquí se presenta se enmarca en el Proyecto de Innovación Educativa "Aplicación de las TICs y el Campus Virtual para el aprendizaje colaborativo" (PIE10-086), financiado por la Universidad de Málaga en el bienio 2010-2012.

\section{Las autoras}

María Jesús Ruiz Muñoz es Doctora por la Universidad de Málaga y profesora en el Departamento de Comunicación Audiovisual y Publicidad de la Universidad de Málaga. Su línea de investigación gira en torno a la Narrativa Audiovisual y a la aplicación de técnicas de aprendizaje colaborativo en el aula para la adquisición de competencias profesionales.

Isabel Ruiz Mora es Doctora por la Universidad de Málaga y profesora en el Departamento de Comunicación Audiovisual y Publicidad de la Universidad de Málaga. Su línea de investigación se centra en la Responsabilidad Social y las Relaciones Públicas, así como en las nuevas metodologías docentes. 